\title{
Pulmonary complications of chemical pneumonia; a case report
}

\author{
Semiha Bahceci Erdem, M.D. ${ }^{a}$, Hikmet T. Nacaroglu, M.D. ${ }^{a}$, Rana Isgüder, M.D. ${ }^{b}$, Canan S. Unsal Karkiner, M.D. ${ }^{a}$, \\ Hüdaver Alper, Professor, M.D. ${ }^{d}$ and Demet Can, Professor, M.D. ${ }^{a}$
}

\begin{abstract}
Hydrocarbon aspiration (HA) can cause significant lung disease by inducing an inflammatory response, hemorrhagic exudative alveolitis, and loss of surfactant function. The most serious side effect of HA is aspiration pneumonia. Pneumothorax, pneumatocele, acute respiratory distress syndrome(ARDS), pulmonary abscess, bronchopleural fistula, bilateral hemorrhagic pleural effusion and pyopneumothorax were previously reported.

Hereby we report a patient hospitalized due to aspiration pneumonia who developed pleurisy and pneumothorax after drinking paint thinner.

It is presented as it was seldom reported in children to cause distinct pulmonary complications. Patients with complaints associated with hydrocarbon poisoning must be fully evaluated. They must not be discharged from the hospital early and must be followed for at least 48 hours even if they don't have respiratory symptoms. It should be kept in mind that severe pulmonary complications can develop in patients with chemical pneumonia.

Key words: aspiration pneumonia, hydrocarbons, poisoning, pleural effusion, pneumothorax.
\end{abstract}

http:/ /dx.doi.org/10.5546/aap.2016.eng.e245

\section{INTRODUCTION}

Hydrocarbons are heterogeneous group of substances that are primarily composed of carbon and hydrogen molecules. They are quite abundant in modern life. Some of the most commonly used hydrocarbons include gasoline, lubricating oil, motor oil, mineral spirits, lighter fluid/naphtha, lamp oil, and kerosene. ${ }^{1}$ Paint thinner ranks among the volatile hydrocarbons group. Acute hydrocarbon poisonings frequently lead to chemical pneumonia and rarely develop pneumatocele, pulmonary abscess, pleural

a. Department of Pediatric Allergy, Dr. Behcet Uz Children's Hospital, Izmir, Turkey.

b. Department of Intensive Care, Dr. Behcet Uz Children's Hospital, Izmir, Turkey.

c. Department of Radiology, Ege University Faculty of Medicine, Izmir, Turkey.

E-mail Address:

Hikmet T. Nacaroglu, M.D.: tekin212@gmail.com

Funding: None.

Conflict of interest: None.

Received: 11-4-2015

Accepted: 1-22-2016 effusion and ARDS. ${ }^{1,2}$ Though hydrocarbon poisonings and subsequent chemical pneumonia are mostly observed in children, not much child cases had been reported previously with pneumonia, pleurisy and pneumothorax. Below is presented the case of severe chemical pneumonia developed along with pleurisy and pneumothorax due to paint thinner consumption.

\section{CASE PRESENTATION}

It was found out that 9 year-old male patient with mental retardation had been hospitalized one week ago in another center and treated with antibiotic following his dyspnea developed after he had drunk thinner inadvertently which was left around by the parents in a glass. He was referred to our hospital since he had neither clinical nor radiological improvement, contrarily; he had an increase in the disease state. There was no characteristic in prenatal and natal history. His family indicated that he had developed mental retardation when he was one month old during his first hospitalization due to febrile convulsions. In his family history, there was no specific feature other than epilepsy in his father. In his physical examination, it was determined that weight and height was $25-50$ percentile, fever: $37.9^{\circ} \mathrm{C}$, pulse: 152 beats/min., respiratory rate: $42 / \mathrm{min}$, arterial blood pressure: $123 / 83 \mathrm{mmHg}, \mathrm{SaO}_{2}: 80 \%$, there were subcostal and intercostal retractions, on auscultation decreased respiratory sounds at the basal zones and bilateral crepitations were present; and other systems' examinations were normal. In the chest radiography, there were bilateral basillary pneumonic infiltrations (Figure 1a). In the thorax ultrasonography, in left pleural cavity a $2.2 \mathrm{~mm}$ and at the right pleural cavity a $13.2 \mathrm{~mm}$ thick effusion were established. At first it was planned that the patient should be monitored with antibiotic treatment, and drainage should be carried out in case of an increase in the effusion. But the respiratory distress suddenly increased on the third day of his hospitalization and it was observed that there was an increase in the effusion and pneumothorax was identified in the chest x-ray (Figure 1b). In thorax CT, pleural effusion reaching $26 \mathrm{~mm}$ in its ultimate thickness which extends up to the apex in the 
right lung, and anterior to the effusion, a wide air density (a pneumothorax) drew attention (Figure 2). Consolidation areas involving air bronchograms in the lower lobe at the right lung, and air densities in the consolidated middle lobe level were determined. Opacities compatible with prevalent consolidation in left lung lingula and lower lobe segments were available. This radiological appearance was evaluated as necrotizing pneumonia. Minimal pleural effusion of 7-mm thickness was observed at the basal of the left lung. Thoracal tube drainage was placed and the fluid obtained was compatible with exudation in feature. Biochemical characteristics and $\mathrm{pH}$ of exudate was not consistent with empyema; and since the culture was negative, empyema was not considered. The patient was followed up with the thorax tube for five days. The tube was removed when the pneumothorax improved. In the follow-up, N-Acetyl cysteine nebule therapy and physiotherapy programme introduced to the patient who developed atelectasia. Our patient recovered satisfactorily following the initial treatment consisting of oxygen inhalation, IV antibiotics, inhaled steroids and salbutamol, and supportive measures without any residual pulmonary sequela. The patient was discharged from the hospital 40 days later when his symptoms got prominently better.

\section{DISCUSSION}

Paint thinner includes a mixture of xylene isomers. Toluene and xylene are aromatic hydrocarbons commonly used as solvent for the manufacturing of pharmaceuticals, paints, and chemicals. ${ }^{1}$ Parents careless use of toxic substances at home keeping them in the bottles like water, fruit juice or carbonated beverages may facilitate the accidental drinking of them by children. Our patient had accidentally drunk the

FIgURE 1A-B. a) Bilateral pneumonic infiltration in chest $X$-ray at the base of lungs $b$ ) Chest $X$-ray revealing pneumothotax.

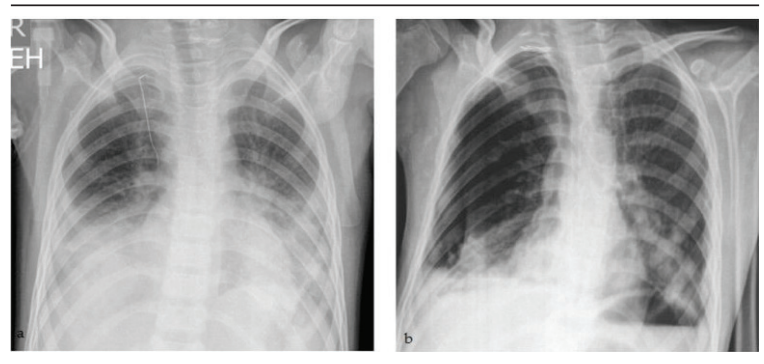

thinner put into a glass by the parents thinking it was water. Poisonings with hydrocarbon compounds rank among the severe childhood poisonings. Chemical pneumonia is the most common complication of hydrocarbon poisonings. Chemical pneumonitis in most of the cases is interstitial and bilateral. ${ }^{2-4}$ The ultimate result of hydrocarbon aspiration is interstitial inflammation, intraalveolar hemorrhage and edema, hyperaemia, bronchial necrosis, and vascular necrosis. 5,6 The hemorrhagic alveolitis and bronchial and vascular necrosis can result in a hemorrhagic pleural effusion, which has been rarely reported. ${ }^{7}$

Following poisoning, respiratory effects occur as a result of aspiration and ingestion leading to lipoid pneumonitis that increases the transpulmonary pressure. Transpulmonary pressure that exceeds the tensile strength of the non-cartilaginous terminal airways and alveolar saccules can damage the respiratory epithelium. Disruption of epithelial integrity permits air to enter the interstitium, causing pulmonary interstitial emphysema. Persistent elevated transpulmonary pressure facilitates the dissection of air toward the visceral pleura and/or hilum via the peribronchial and perivascular spaces. Rupture of pleural surface allows the adventitial air to decompress into pleural space causing pneumothorax. ${ }^{8}$

Rare pulmonary complications include the development of a pneumothorax, pneumatocele, pyopneumothorax, ARDS or bronchopleural fistula. ${ }^{8-10}$ Few cases of kerosene poisoning-

FIGURE 2. Thoracal CT image showing pleural effusion, and pneumothorax anterior to the effusion.

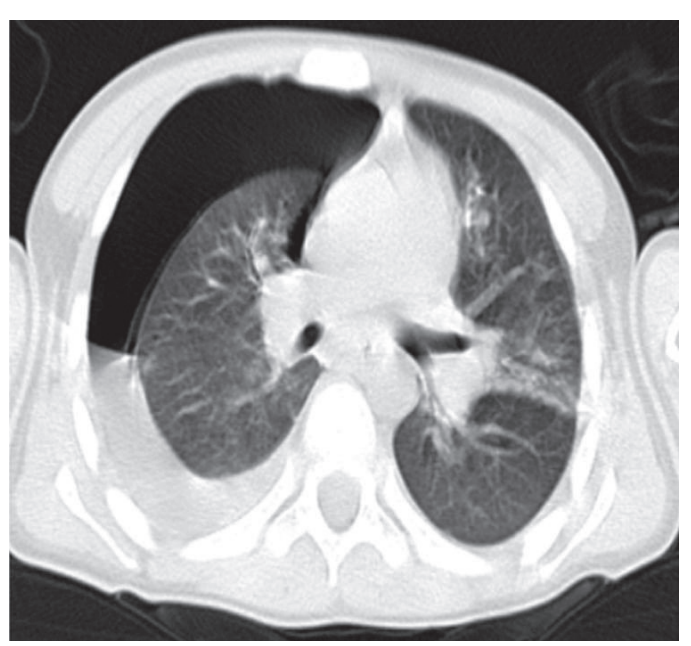


induced pneumothorax and empyema have been reported in literature but pyopneumothorax is a rare entity. ${ }^{4,8}$ Lifshitz et al., ${ }^{5}$ carried out a 4 -year follow up study between 1995-1999 and reported that 118 (43\%) out of 274 cases with hydrocarbon poisonings had pneumonia, hypoxia and fever; pneumonia was interstitial and bilateral; and it was associated with vomiting. ${ }^{4}$ Pleurisy and pneumothorax were not reported in these cases. Prasad et al., ${ }^{10}$ reported a 40 year old patient with bilateral hemorrhagic pleurisy and pneumonia developed depending on paraffin oil consumption in 2011, pleurisy was hemorrhagic and of exudative character, however, the culture was negative. Pneumothorax did not accompany the incident in this patient, as well. Chaudhary et al., ${ }^{8}$ reported that an adult patient developing pyopneumotorax following paraffin oil intake had Staphylococcus aureus reproduction in thoracentesis fluid; and the patient recovered with thoracal tube, antibiotic treatment and pleural decortication. However in our patient, pneumonia, pleurisy and pneumothorax developed simultaneously. Radiographic findings seen in hydrocarbon poisonings include unilateral or bilateral lung consolidation, well-defined nodules, pneumatoceles (well-defined cavitary nodules), pleural effusion, and spontaneous pneumothorax, and many of these findings were observed in our patient.

Induced vomiting is not recommended in the treatment of any hydrocarbon derivatives. Inhaled oxygen is helpful in pulmonary aspiration. B2-agonists are useful in the presence of bronchospasm. Invasive or non-invasive mechanical ventilation (PEEP and CPAP) can be required, nevertheless, monitoring for pneumatocele and pneumothorax should be carried out. If there is an increase in leukocytosis and infiltration findings as well as fever after the first 24-48 hours, antibiotic treatment should be added owing to secondary bacterial pneumonia risk. ${ }^{3-10}$ Thoracal tube drainage was carried out for spontaneous pneumothorax and pleural effusion in our case. Our patient recovered satisfactorily following the initial treatment consisting of oxygen inhalation, antibiotics, inhaled steroids and salbutamol, and supportive measures without any residual pulmonary sequela.

In conclusion, our patient is one of the rare pediatric cases according to the literature that pleurisy and pneumothorax association was observed following chemical pneumonia developed due to hydrocarbonated compound ingestion. ${ }^{11,12}$ Patients with complaints associated with hydrocarbon poisoning must be fully evaluated. They must not be discharged from the hospital early and must be followed for at least 48 hours even if they don't have respiratory symptoms. It should be kept in mind that severe pulmonary complications can develop in patients with chemical pneumonia.

Owing to inappropriate storage conditions of hydrocarbons in the household, accidental ingestion of even small amounts of them comprises serious childhood poisonings. Although most of the children improves without any sequelae or complication, some may die due to respiratory failure. Hydrocarbon compounds should be kept out of reach of children in their original packages under safe covers.

\section{REFERENCES}

1. Bronstein AC, Spyker DA, Cantilena LR Jr, Green J, et al. 2006 Annual Report of the American Association of Poison Control Centers' National Poison Data System (NPDS). Clin Toxicol (Phila) 2007;45(8):815-917.

2. Argo A, Bongiorno D, Bonifacio A, Pernice V, et al. A fatal case of a paint thinner ingestion: comparison between toxicological and histological findings. Am J Forensic Med Pathol 2010;31(2):186-91.

3. Haas C, Lebas FX, Le Jeunne C, Lowenstein W, et al. Les pneumopathies par inhalation d'hydrocarbures. À propos de trois observations. Ann Med Interne (Paris) 2000;151(6):438-47.

4. Osterhoudt KC, Ewald MB, Shannon M, Henretig FM. Toxicologic emergencies. En: Fleisher GR, Ludwig S, ed. Textbook of Pediatric Emergency Medicine. 6th ed. Philadelphia: Lippincott Williams \& Wilkins; 2010:1171221.

5. Lifshitz M, SoferS, Gorodischer R. Hydrocarbon poisoning in children: a 5-year retrospective study. Wilderness Environ Med 2003;14(2):78-82.

6. Dyer KS. Hydrocarbons. En:Wolfson AB, Hendey GW, Ling LJ, Rosen CL, et al, ed. Harwood-Nuss' Clinical Practice of Emergency Medicine. 4th ed. Philadelphia: Lippincott Williams and Wilkins; 2005.P.1590-3.

7. Mylonaki E' Voutsas V, Antoniou D, Papakosta D, et al. Hydrocarbon pneumonitis following liquid paraffin aspiration during a fire-eating performance: a case report. J Med Case Rep 2008;2:214.

8. Chaudhary SC, Sawlani KK, Yathish BE, Singh A, et al. Pyopneumothorax following kerosene poisoning. Toxicol Int 2014;21(1):112-4.

9. Domej W, Mitterhammer H, Stauber R, Kaufmann P, et al. Successful outcome after intravenous gasoline injection. $J$ Med Toxicol 2007;3(4):173-7.

10. Prasad R, Karmakar S, Sodhi R, Karmakar S. Bilateral hemorrhagic pleural effusion due to kerosene aspiration. Lung India 2011;28(2):130-2.

11. Marandian MH, Sabouri M, Youssefian H, Behvad A, et al. [Pneumatoceles and pneumothorax following accidental hydrocarbon ingestion in children. A study of 50 cases in Iran]. Ann Pediatr (Paris) 1981;28(9):687-91.

12. Majeed HA, Bassyouni H,Kalaawy M, FarwanaS. Kerosene poisoning in children: a clinico-radiological study of 205 cases. Ann Trop Paediatr 1981;1(2):123-30. 